DI

DESY 94-050

$$
\begin{aligned}
& \text { IESY } 94.050 \\
& \text { = (2) } 0415
\end{aligned}
$$

BAIKAL 93-16

March 1994

\title{
Track Reconstruction and Background Rejection in the Baikal Neutrino Telescope
}

\author{
presented by C. Spiering \\ Deutsches Elektronen-Synchrotron DESY \\ Institut für Hochenergiephysik IfH, Zeuthen
}

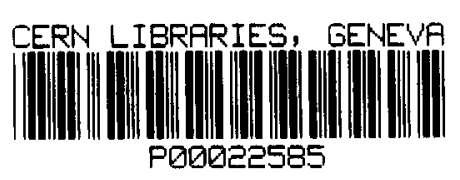

ISSN 0418-9833 


\title{
Track Reconstruction and Background Rejection in the Baikal Neutrino Telescope
}

\author{
I.A.Belolaptikov ${ }^{1}$, J.-A.M.Djilkibaev ${ }^{2}$, S.I Klimushin ${ }^{2}$, \\ J.Krabi $^{5}$, O.Ju.Lanin ${ }^{2}$, N.Hasselmann ${ }^{5}$, E.A.Osipova ${ }^{3}$, \\ A.A.Pavlov ${ }^{4}$, Ch.Spiering ${ }^{5}$, R.Wischnewski ${ }^{5}$ \\ presented by $\mathrm{CH}$.SPIERING \\ 1 Joint Institute for Nuclear Research (Dubna, Russia), ${ }^{2}$ Institute for \\ Nuclear Research of the Russian Academy of Science (Moscow, Russia), \\ ${ }^{3}$ Moscow State University (Moscow, Russia), ${ }^{4}$ Irkutsk State University \\ (Irkutsk, Russia), ${ }^{5}$ DESY-Institut für Hochenergiephysik (Zeuthen, \\ Germany)
}

\begin{abstract}
We describe procedures for reconstructing muon tracks in the BAIKAL Neutrino Telescope, including filtering out badly reconstructed events. Special attention is paid to rejecting those downward going muons which fake upward going muons from neutrino interactions. It is shown that a suppression factor of $10^{6}$ - necessary to operate an underwater neutrino telescope at $1100 \mathrm{~m}$ depth - can be achieved with an array consisting of 200 photomultipliers. We present first results from NT-36, an array of 36 PMTs deployed in April 1993. We observe satisfactory agreement between Monte Carlo results and experimental data, providing confidence that our simulations of the full detector are indeed realistic.
\end{abstract}

to be published in Proc. 3nd NESTOR Int. Workshop, Oct. 1993, Pylos, Greece 


\section{Introduction}

The reconstruction of the track parameters of a particle crossing a deep underwater detector turns out to be complicated compared to the same task in typical underground detectors. In tracking devices like MACRO the track is well defined by the coordinates of scintillators or streamer tubes. In Cherenkov detectors like KAMIOKANDE the track is given by hit patterns and arrival times of photomultipliers (PMTs) covering the inner surface of the water volume with a rather fine lattice. In the case of deep underwater arrays, a lattice of PMTs is spanned over the telescope volume with a comparatively large spacing. The large spacing is chosen in order to maximize - for a given number of PMTs - the effective area, on the other hand it results in a lower degree of redundancy compared with most underground detectors.

For many years, the investigations of track reconstruction with underwater detectors were restricted to Monte-Carlo generated events. Although small prototype detectors consisting of a few PMTs had been deployed in earlier years by the BAIKAL collaboration $[1,2,3]$, the DUMAND collaboration [4] and the NESTOR collaboration $[5,6]$, none of these detectors allowed a full spatial reconstruction of muon tracks.

Since April 1993, a detector consisting of 36 PMTs attached to three strings is operated in lake Baikal $[7,8]$. The detector, christened NT-36, is the first underwater array allowing full three-dimensional reconstruction of muons. It gives the first possibility to check numerous methods of track reconstruction and background rejection developed by our collaboration. The crucial question in using an underwater detector as a neutrino telescope is whether one can reject the background of downward atmospheric muons faking upward moving muons. At $1 \mathrm{~km}$ depth, downward muons exceed the upward moving muons from interactions of atmospheric neutrinos by a factor of $10^{6}$. The expected median angular resolutions for planned deep underwater detectors like the Baikal telescope NT-200 [912], DUMAND-II [13-15], AMANDA [16-19] and NESTOR [5,6] are between $0.5^{\circ}$ and $2^{\circ}$. However, the zenith mismatch angle distribution from a simple fit alone is characterized by a long tail. Following only the usual fit procedure, the number of fake events in NT-200 would exceed the number of events due to neutrinos generated in the atmosphere by several orders of magnitude.

This report describes algorithms to remove badly reconstructed events while accepting most of the events reconstructed with mismatch angles less than $5^{\circ}$. We show that a rejection factor of $10^{6}-$ necessary to operate a neutrino telescope at a depth of 1100 m.w.e. - can be obtained in 
NT-200 over the full lower hemisphere. Atmospheric neutrinos could be investigated over a low background in a cone of $70-80^{\circ}$ around the opposite zenith.

\section{The Detector}

The NT-200 array will consist of a total of 192 optical modules (OMs) positioned along 8 vertical strings - one central string surrounded by 7 outer strings [11]. The OMs are grouped in pairs along each of the strings, alternatively looking upward and downward. Thus, NT-200 has a symmetric response to muons coming from the upper hemisphere and the lower hemisphere. This symmetry leads to a good reconstruction accuracy for downward muons, essential for effective rejection of these muons when asking for upward muons generated in neutrino interactions. The distance between pairs looking face to face is $7.5 \mathrm{~m}$, while pairs looking back to back are $5 \mathrm{~m}$ from each other. The two PMTs of a pair are connected in coincidence and define, in our notation, a channel. The muon trigger requires a certain minimum number (3-6) of fired channels within a coincidence window of $500 \mathrm{nsec}$. For each of the fired channels $i$, the following data are available at the shore: a) the coordinates $x_{i}, y_{i}, z_{i}$, b) the signal arrival time $t_{i}$ of the one of the two PMTs that has fired first and c) the summed charge $a_{i}$ from both PMTs.

NT-36 consists of 36 PMTs arranged at the central and two neighbouring outer strings attached to the heptagonal frame which will later carry NT-200 (see [8]). The arms of the frame were inclined, decreasing the distance between central and outer strings from $21.5 \mathrm{~m}$ (NT-200) to 15.5 $\mathrm{m}$. The 36 PMTs define 18 channels, i.e. a lattice of 18 space points.

\section{Reconstruction Procedures and Appli- cation to NT-200}

The model underlaying the reconstruction procedure is that of a single muon track. This, of course, is a strong simplification since in reality, and also in our Monte Carlo event generator, stochastic energy loss mechanisms yielding electromagnetic cascades (pair generation, bremsstrahlung, nuclear interactions) play an essential role. Moreover, muons often appear not as single muons but in bundles of nearly parallel muons. Anyway, the first attempt to analyse muons crossing the array is to fit them with a single muon parametrization. 
The parameters of a single muon track can be determined by minimizing the function

$$
S_{t}^{2}=\sum_{i=1}^{N_{\text {his }}}\left(T_{i}\left(\theta, \phi, u_{0}, v_{0}, t_{0}\right)-t_{i}\right)^{2} / \sigma_{t_{i}}^{2}
$$

Here, $t_{i}$ are the experimental times and $T_{i}$ the "theoretical" times expected for a given set of track parameters. $N_{h i t}$ is the number of hit PMT pairs, $\sigma_{t i}$ are the time errors. The five parameters defining a straight track are: $\theta$ and $\phi$ - the zenith and azimuth angle of the muon track, respectively, $u_{0}$ and $v_{0}$ - the two coordinates of the track point nearest to the detector center on a plane perpendicular to the track, and $t_{0}$ - the time the muon passes that point.

Other parametrizations are possible, for instance one may define the space coordinates in the (fixed) detector system instead in a system attached to the muon track $[21,22]$, or replace the time parameter by a third spatial coordinate $z_{0}[24]$. Furthermore, by setting $\vartheta S_{t}^{2} / \vartheta t_{0}=0$ and solving for $t_{0}$, an analytical solution for the time parameter can be found [23]. This reduces the parameter space to be searched for a $\chi^{2}$ minimum from five to four dimensions. Presently, we are checking the pro's and con's of the different approaches with respect to accuracy, robustness, fake event rate and speed.

Due to the large statistical fluctuations of few-photoelectron amplitudes, and since the expected amplitude from high energy muons is determined by highly stochastical energy loss processes [11], we did not include the amplitude term

$$
S_{a}^{2}=\sum_{i=1}^{N_{h i t}}\left(A_{i}\left(\theta, \phi, u_{0}, v_{0}, t_{0}\right)-a_{i}\right)^{2} / \sigma_{a_{i}}^{2} .
$$

into the fit procedure.

$B y$ various reasons, the result of the fit deviates from the true parameters of the trajectory in most cases. Firstly, the $S_{t}^{2}$ function has a complicated behaviour, characterized in many cases by several local minima. Secondly, there are often several true minima reflecting internal symmetries of the detector. These ambiguities are not resolvable by time information alone, and amplitude criteria turn out to be extremely important in order to decide which of the minima is most realistic. Thirdly, it often happens that a large fraction of the hit PMTs detect only light from showers and not from the muon itself. Since photons from electromagnetic showers are generally delayed compared to directly emitted 
photons, and since they have a different angular distribution, the picture of a naked muon fails. The situation may be further complicated by photons delayed due to light scattering in water.

We adopted the following reconstruction procedure:

- preliminary analysis including several causality criteria and a 0th approximation,

- $\chi^{2}$ minimum search,

- application of criteria to reject badly reconstructed events.

The causality critera are applied in order to ensure that the hits are due to a particle moving with the velocity of light and emitting light under the Cherenkov angle $\eta$. The simplest of the criteria tested,

$$
\left|t_{i}-t_{j}\right| \cdot \cos \eta \leq\left|\vec{r}_{i}-\vec{r}_{j}\right|
$$

is applied to all combinations of fired channels. $t_{i}, t_{j}$ and $\vec{r}_{i}, \vec{r}_{j}$ are the times of response and the positions of the channels. Events containing combinations strongly violating (3) are rejected. Actually, one need not necessarily reject the whole event but only that channel (those channels) causing violations of (3). In ocean detectors operating the PMTs in single mode instead as pairs in coincidence, hits from $K^{40}$ signals and PMT noise (typically $50-100 \mathrm{kHz}$ ) are mixed into practically every event [24-26]. These hits have to be eliminated by various criteria like (3) and by repeated fitting procedures identifiying the PMTs with the highest time residuals (see below). The situation is different for detectors like AMANDA and BAIKAL, where the percentage of hits not related to the muon is small. AMANDA profits from the low noise rate of the PMTs and from the sterile medium surrounding the PMTs, BAIKAL from the pairwise coincidence reducing the counting rate of a channel to typically $200 \mathrm{~Hz}$. For the NT-200 detector, the average number of hits not due to Cherenkov light is only 0.03 /event, for DUMAND-II it is about 13 /event $[20]^{1}$.

After having applied the causality criteria, a 0 th approximation for the zenith angle, $\theta_{o}$, and the azimuth angle, $\phi_{o}$, is performed. To determine $\phi_{o}$, we define an "entrance" and an "exit" string in the following way: The strings are ordered according to the time of the earliest firing pair along the string. Then, the entrance string is the first and the exit string the

\footnotetext{
${ }^{1}$ Actually, this high rate of noise hits seems to result in fake event rates similar to BAIKAL or AMANDA $[25,26]$.
} 
last of all strings. $\phi_{o}$ is defined as the angle between the plane spanned by the entrance and the exit string and the $x, z$-plane.

$\theta_{0}$ is obtained in two ways. If one of the strings includes 4 or more fired PMT pairs, $\theta_{0}$ is obtained by an analytic solution of the equation of muon motion for these pairs. If there is no string with at least 4 firing pairs, a plane wave approximation as proposed in [24] is applied.

For small arrays like NT-36, the plane wave approximation turned out to be superiour for the $\theta_{0}$ as well as the $\phi_{0}$ determination.

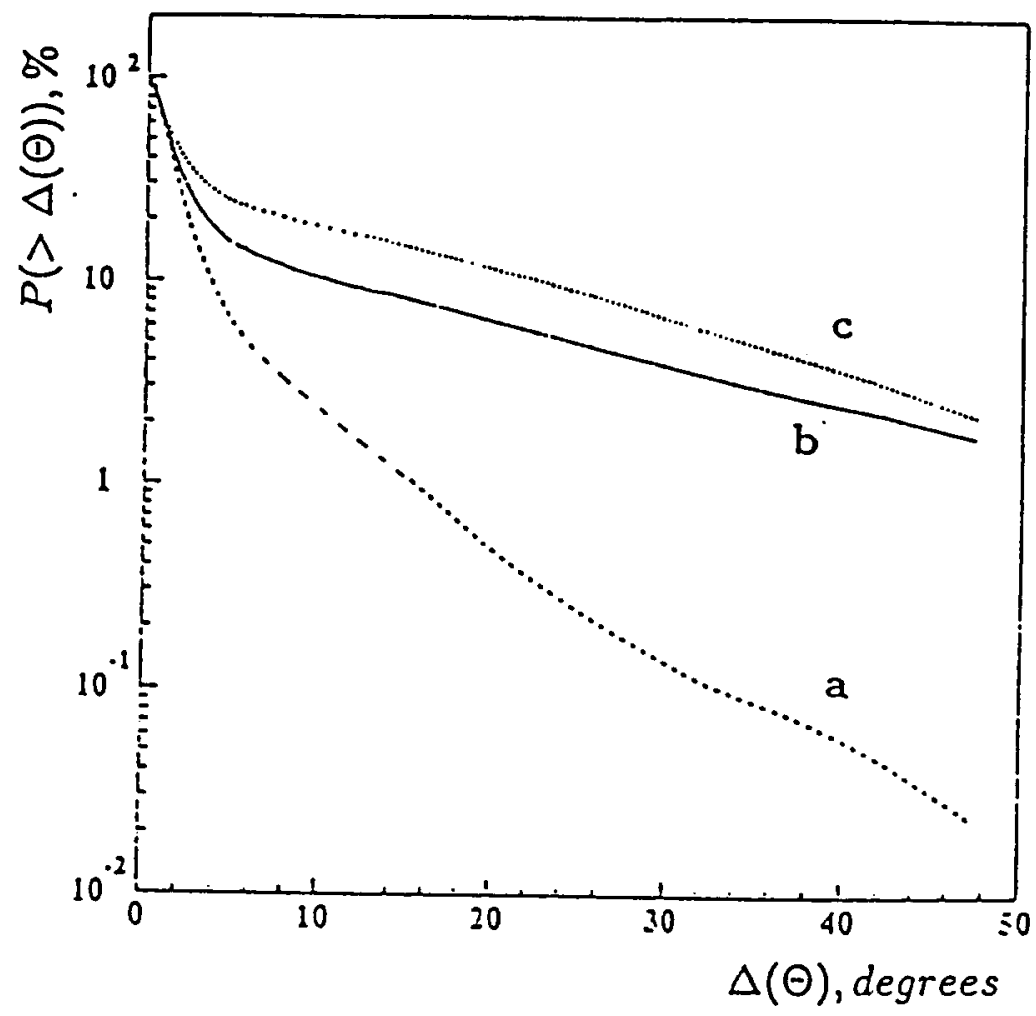

Fig.1: Distribution of the zenith mismatch angle $\Delta \theta$ in NT-200 after $\chi^{2}$ search. a) $M C$ parameters taken as starting values, b) starting values chosen as described in the text, c) "bad" starting values (see also text).

Fig. 1 demonstrates the influence of the 0th approximation on the result of the subsequent $\chi^{2}$ fit. It shows the zenith mismatch angle, $\Delta \theta$, obtained from the $\chi^{2}$ fit. The input for the main fit is taken as a) the precise Monte Carlo generated value, b) the values given by our procedure for the 0th approximation and c) by an approximation using only the positions of the uppermost and the lowest of the fired channels for a $\theta_{0}$ approximation and choosing all other starting values arbitrarily. As 
can be seen, the "bad" 0th approximation (c) gives twice the number of badly reconstructed events $\left(\Delta \theta \geq 5^{\circ}\right)$ compared with the "good" approximation (b). Due to the complicated structure of the $\chi^{2}$ landscape, case (b) yields significantly worse results than the "ideal" case (a), where exactly the generated parameter values have been taken as the 0 th approximation for the fit. Stochastic processes, time jitters of the PMTs and the electronics together with the strong non-linearity of the problem cause a long tail in the $\Delta \theta$ distribution even for the latter case.

Using the results from the 0 th approximation as starting values, a gradient $\chi^{2}$ search is performed. If after 30 steps no convergence has been reached, the search is interrupted and the event rejected. For NT-200, about 95 percent of the events pass this condition.

After the fit, various criteria are applied to identify badly reconstructed events. These events may then be rejected, fitted repeatedly with other starting values etc. The toolset of these criteria involves the following cuts:

i) upper limit on the minimum $\chi^{2}$ found;

(This limit, of course, depends on the actual number of hits, i.e., on the degrees of freedom.)

ii) upper limit on the time residuals of each individual PMT ${ }^{2}$;

(In the case of experiments with an average number of accidental hits of order of unity or larger, there will be PMTs with correspondingly large time residuals in practically every event. The PMT with the largest residual would be rejected and the fit repeated, eliminating in this way subsequently most of the accidentals [24,25].)

iii) upper limit on the distance of each PMT (or at least the nearest $\mathrm{PMT}$ ) to the reconstructed track;

iv) lower limit on the product of probabilities, $P_{\text {nohit }}$, of non-fired PMTs not to respond to a naked muon;

v) rejection of "quasi-peripheral" events, i.e., events with a reconstructed track crossing the array volume but not intersecting the polygon spanned by the fired PMTs;

vi) upper limit on the error matrix eigenvalues (large semi-axes of error ellipsoids) as obtained from the fit;

\footnotetext{
${ }^{2}$ Referring to the BAIKAL experiment, "PMT" always should be replaced by "channel" or "PMT pair".
} 
vii) upper limit on the angular error $\sigma_{\theta}$ obtained from the error matrix;

viii) lower limit on the planarity, i.e., rejection of events where all PMTs are in a plane;

ix) lower limit on the main axis of the tensor of inertion $I$ of the PMTs hit $\left(I=\sum a_{i}\left(r_{i}^{2}-\left(\vec{r}_{i} \cdot \vec{n}\right)^{2}\right)\right.$, where $a_{i}$ are the amplitudes of the hit PMTs and $\vec{r}_{i}$ the radius vectors of the PMTs with respect to their amplitude-weighted center of gravity. $\vec{n}$ defines the axis of rotation.);

(The latter two criteria reject candidates for ambiguities. Criterion viii) relates to left-right ambiguities with respect to a plane and criterion ix) to azimuthal ambiguities with respect to the rotation axis.)

$x$ ) exclusion of zero correlation or full correlation between fitted track parameters.

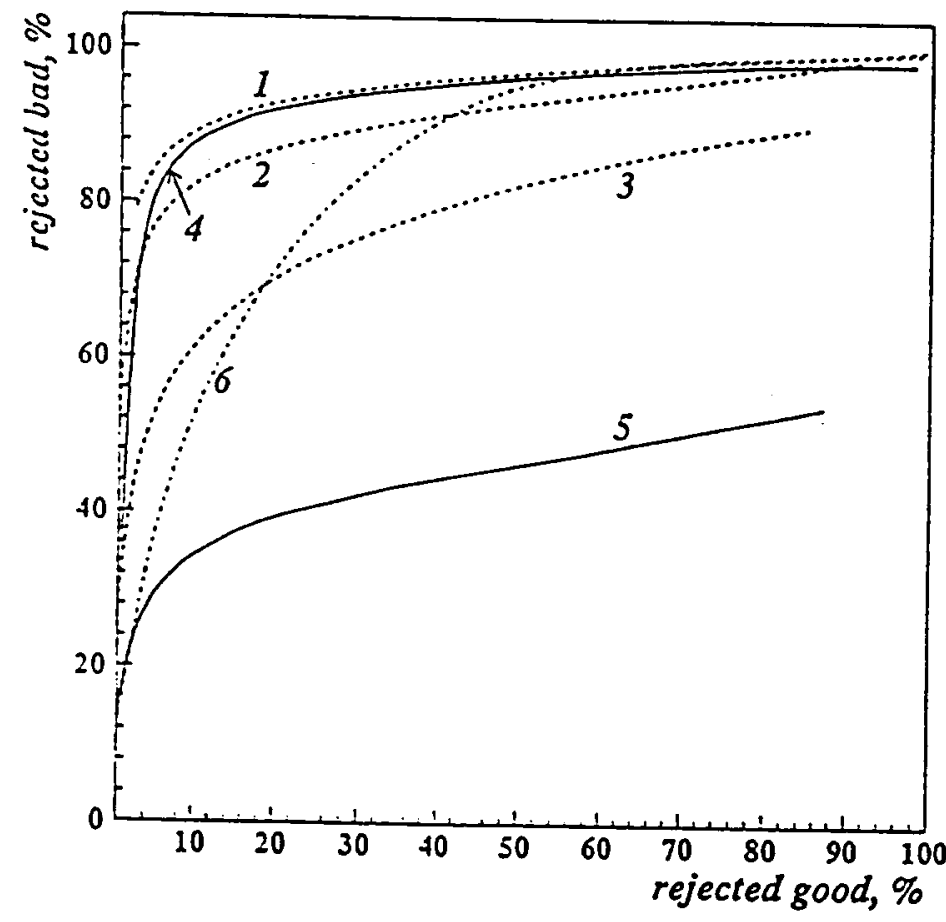

Fig.2: Separation power of different criteria (NT-200):

$1,2,3-\chi^{2}$ criterion for 11,9 and 7 hits, respectively,

$4-P_{\text {nohit-criterion, track inside detector, }}$

$5-P_{\text {nohit-criterion, track outside detector, }}$

6 - largest eigenvalue (large semi-axis of error ellipse) in variables $t_{0}, u_{0}, v_{0}$. 
Fig.2 demonstrates the power of different criteria to separate "good" $\left(\Delta \theta<10^{\circ}\right)$ and "bad" events $\left(\Delta \theta>20^{\circ}\right)$. The parameter along the curves is the relevant cut variable $\left(\chi^{2}, P_{\text {nohit }}\right.$ etc.). The steeper the curve and the higher with respect to the ordinate the kink, the more effectively is the criterion in separating well reconstructed from badly reconstructed events.

We have generated and analyzed about $4 \cdot 10^{6}$ events from single atmospheric muons in NT-200 [27]. No event was found which passed a subset of the criteria listed above $(i, i v, v, v i, v i i, x)$ and was reconstructed as an upward event, corresponding to a preliminary upper limit of about $3 \cdot 10^{2}$ for the number of fake events per year (Fig.3). This compares with about 250 events per year passing all criteria which are due to atmospheric neutrinos from the lower hemisphere. Most of the fake events are expected to concentrate near the horizon. If one selects muons only within a certain cone around the opposite zenith, the signal-to-background ratio should be even better.

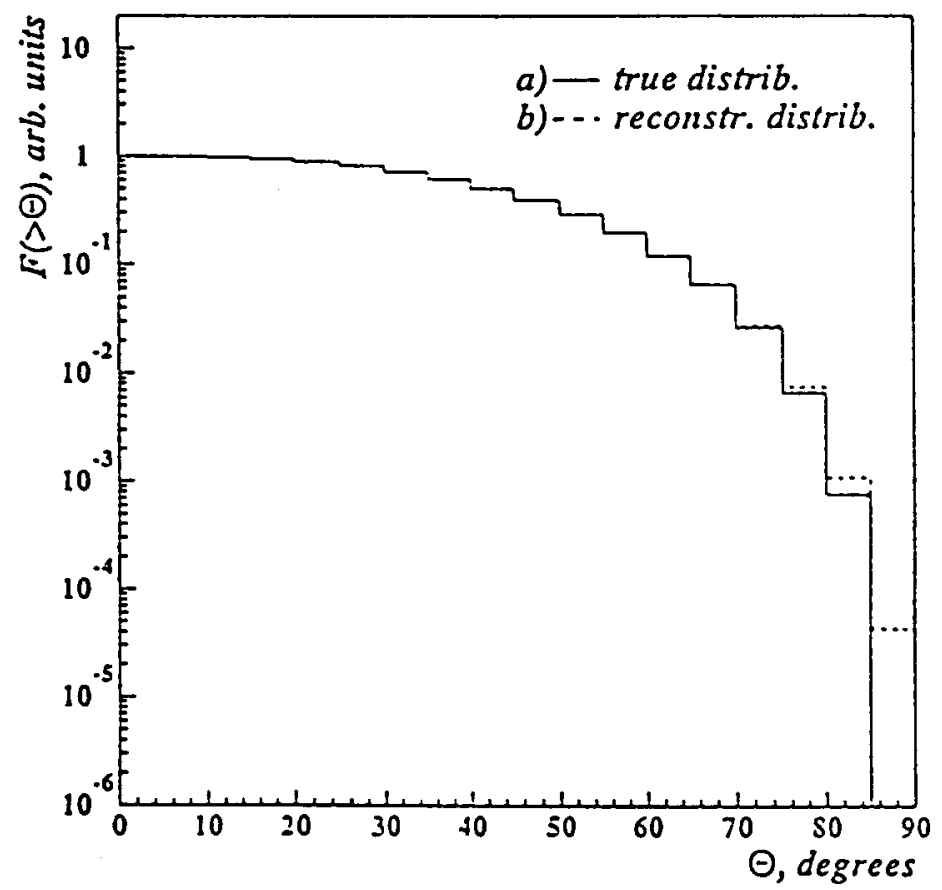

Fig.3: The integral distribution of atmospheric muons in NT-200 as a function of zenith angle $\theta$. (a) generated by Monte Carlo, (b) the same sample after reconstruction procedure.

The results described have been obtained for single muons with the energy and angular distribution expected at $1070 \mathrm{~m}$ depth. In contrast to the simulations of NT-36 (sect.4), muon bundles have not been taken into account. The calculations will be refined with respect to bundles. Also, 
we will improve the robustness of some criteria with respect to changes of the detector geometry (e.g. failing modules), variations of water parameters etc. Neural networks are planned to be used to determine the cuts in the variables mentioned above.

\section{First Results from NT-36}

In the following, we present results obtained with NT-36 (see [8]). The 36 PMTs of the array define a lattice of 18 space points ("channels"). It turned out that some of the criteria developed for NT-200 seem not to be very effective for such small arrays, therefore we have used only criteria $i$-iv, ix and $x$ up to now.

Fig.4 shows the time residual of channel 7 after the fit. Good agreement between $M C$ calculations and experiment is observed if in addition to single muons also muon bundles are included in the MC calculations [30].

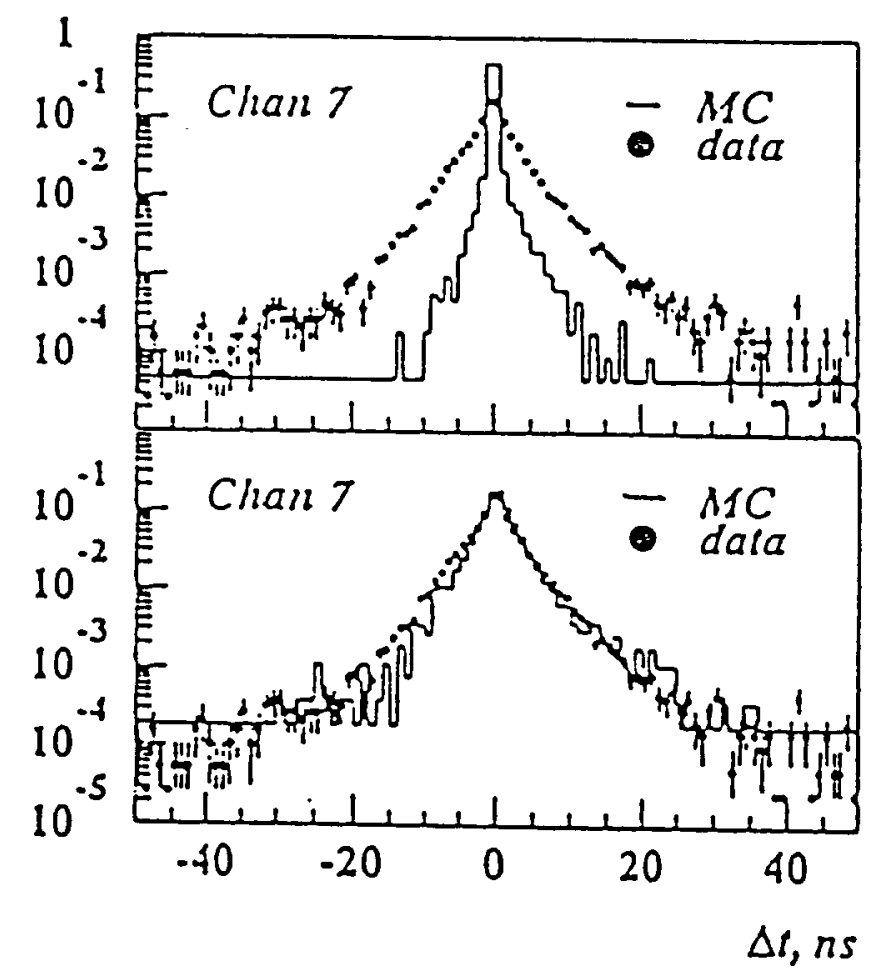

Fig.4: Time residual after fit for channel 7 of NT-36. Points are the experimental data, lines are $M C$ results with including only single muons (top) and including also muon bundles (bottom). 
Actually, the average number of muons per event giving at least one hit is expected from MC estimates to be 1.6 for NT-36. The average number of muons per events strongly depends on the number of pairs hit. One gets an average $1.2,2.0$ and 3.2 muons for 6,10 and 15 hits, respectively (see fig.5). If one requires a high number of hits, one gets a sample of events enriched with multi-muon events. Since multi-muon events do not correspond to the model underlying the $\chi^{2}$-procedure, most of them are rejected by the criteria applied after the fit.

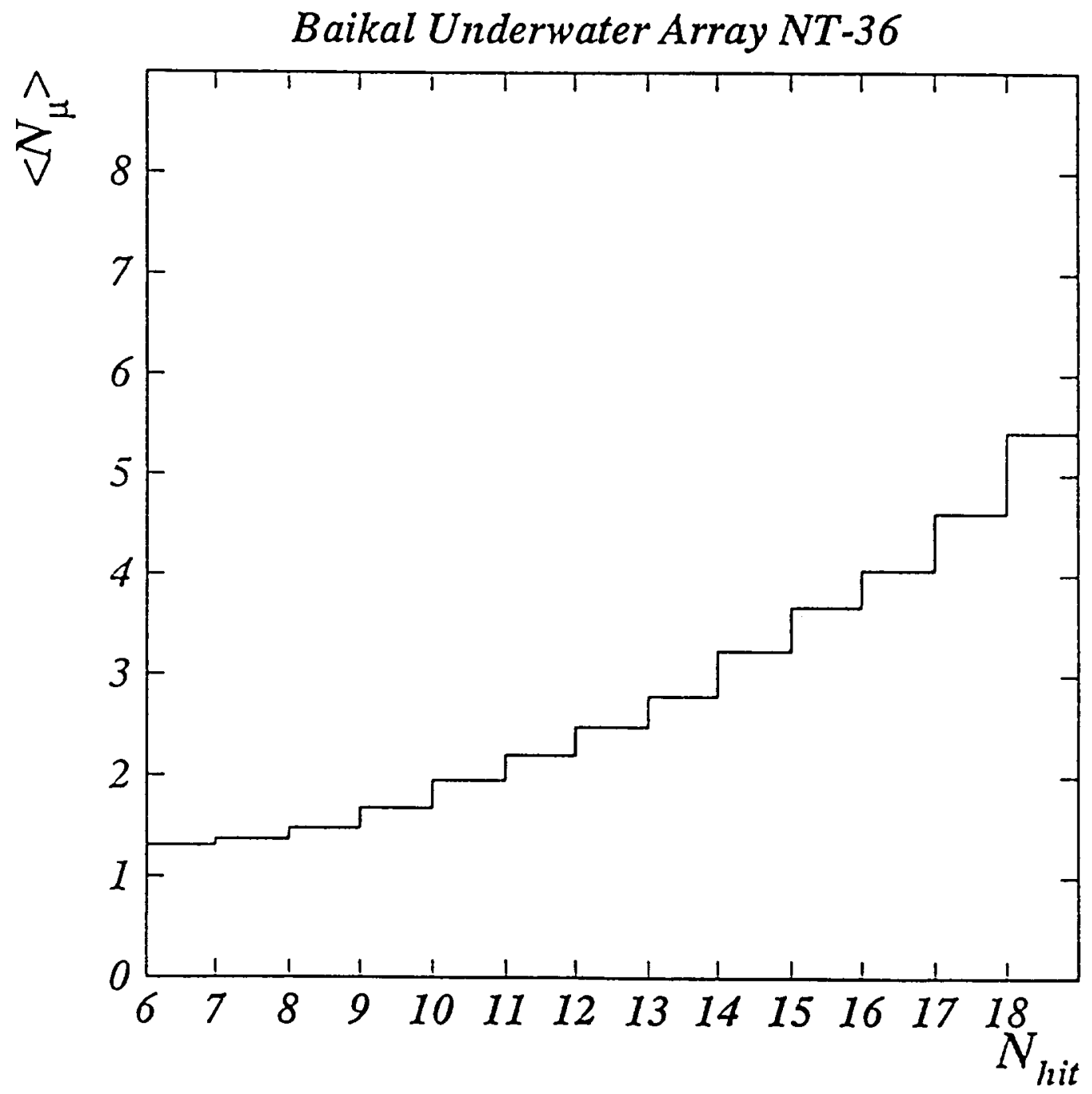

Fig.5: Expected average number of muons per event in NT-36 as a function of the number of hits. A muon is regarded to contribute to the event if at least one of the channels is fired by this muon. 
Fig. 6 shows the distribution of the zenith mismatch angle as obtained from MC calculations. Note that the long tail with large mismatch angles is substantially lowered by application of the rejection criteria. The medium zenith mismatch angle is $5.1^{\circ}$ before and $2.3^{\circ}$ after application of the rejection criteria. The corresponding errors for the full spatial mismatch angle are much larger: $12.5^{\circ}$ and $4.8^{\circ}$, respectively. This is due to the fact that NT- 36 has only 3 strings and that these strings are very close to each other.

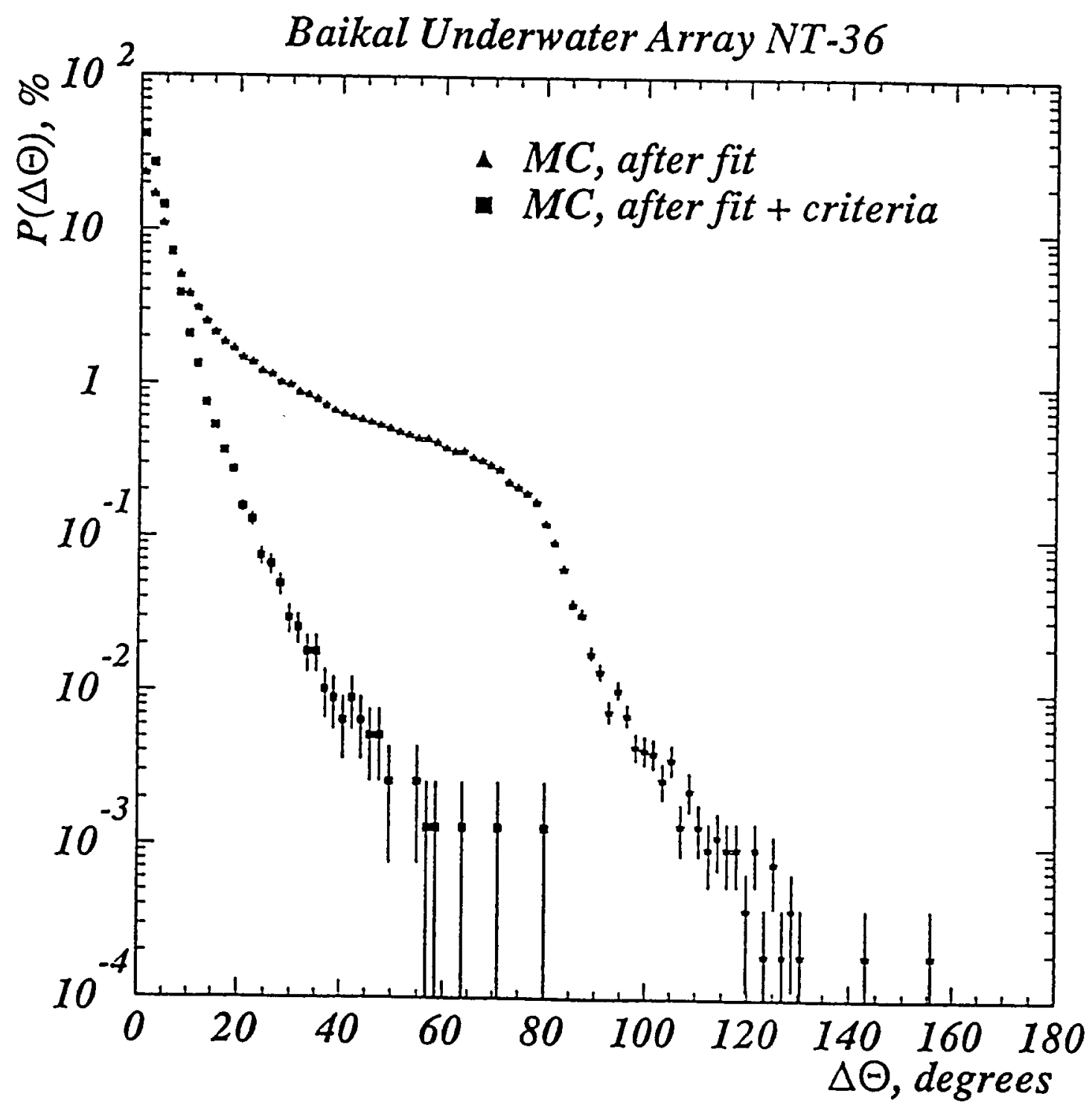

Fig.6: Distribution of the zenith mismatch angle for NT-36 obtained from MC simulations. 
In fig.7, the zenith angle distribution of the reconstructed muons having passed all the cuts is shown. There remains a portion of $10^{-3}$ of the initial sample which is reconstructed as upward going muons. Applying the same cuts to a $\mathrm{MC}$ sample of atmospheric neutrino events from the lower hemisphere one gets a reduction factor of 0.45 . Thus the ratio of fake events to muons from atmospheric neutrinos is $10^{6} \cdot 10^{-3} / 0.45 \approx 2 \cdot 10^{3}$.

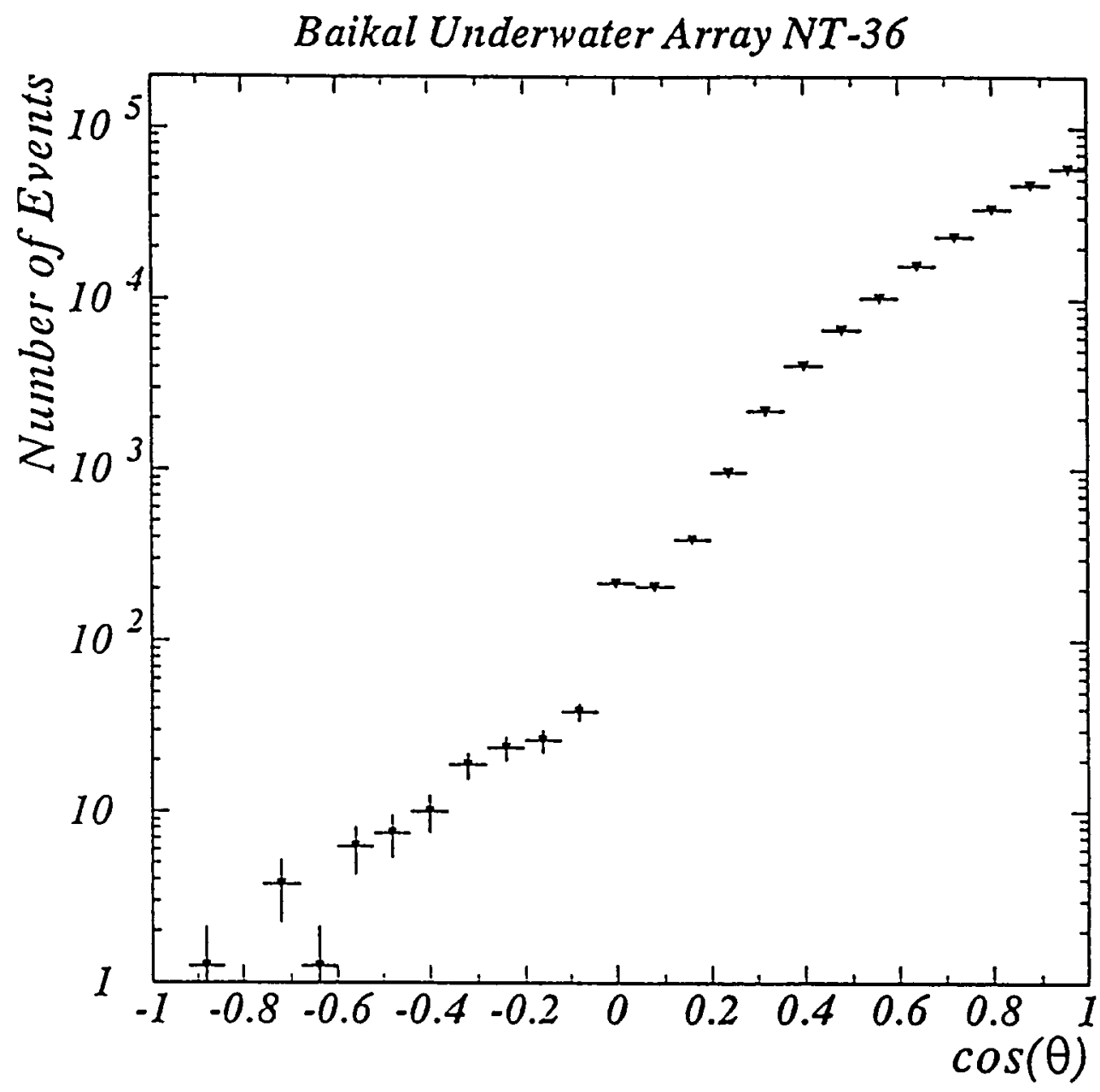

Fig.7: Zenith angle distribution of reconstructed muons in NT-36 after application of rejection criteria.

All the cuts mentioned above basically reject events being candidates for large errors in the fitted parameters. They do not relate to the hemisphere which the muon comes from. Therefore, we finally applied a filter in the space of time differences between modules along one string which essentially acts as a "smooth" cut in the zenith angle [28]. The filter works as follows: 
a)
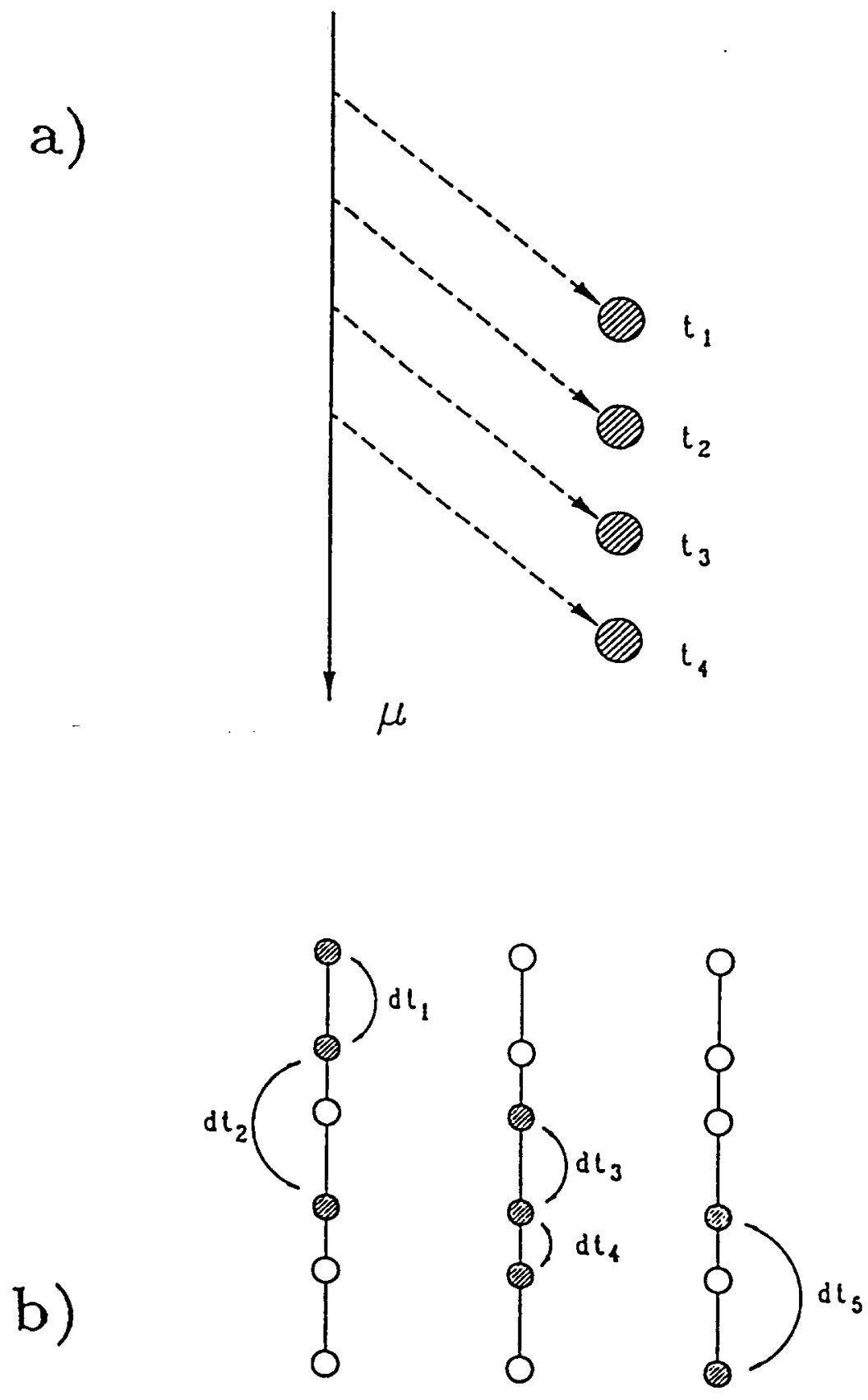

Fig.8: a) Time pattern for a vertically downward moving "naked" muon. b) Definition of the normalized time differences. 
For vertically downward moving particles, the response times along a string are expected to rise monotonously going from the top PMT to the lowest PMT (fig. 8a). This picture, however, is too simplified to describe muons coming from all over the upper hemisphere and generating Cherenkov light not only directly but also via electromagnetic cascades. Therefore, the time differences between all neighbouring fired channels along each of the 3 strings were investigated. Two fired channels separated by silent channels are also counted as neighbours (see fig.8b). In order to allow a comparison, all differences $d t_{i}$ were normalized to a common distance. Then, minimum and maximum $d t_{i}$ were plotted against each other.

Fig.9 shows the $d t_{\max }$-vs.- $d t_{\min }$ plot for (a) the experimental data, (b) the MC events from atmospheric muons and (c) the MC neutrino events. As can be seen, (a) and (b) populate the same region of the phase space (mostly positive $d t$ ), whereas the neutrino events are in the region of negative $d t$. With a very simple cut (requiring $d t_{\max } \leq 5 \mathrm{nsec}$ and $d t_{\min } \leq-20 \mathrm{nsec}$ for neutrino events, and regarding all other events as muonic background) one rejects most muons from the upper hemisphere and cuts away only a few of the neutrino events just below horizon. Applied in conjunction with the standard criteria we found an overall rejection factor of $3 \cdot 10^{-5}$ for our experimental sample, approximately the same as for the sample of MC generated downward muon events, while the survival rate for the $\mathrm{MC}$ neutrino sample was 30 percent. This yields $10^{6} \cdot 3 \cdot 10^{-5} / 0.3 \approx 10^{2}$ as the ratio of fake events to true neutrino events.

It turned out that by careful inspection of the remaining candidates for neutrino events further fake events can be rejected. However, the remaining two orders of magnitude which are necessary to bring the fake events to the same level as the events from atmospheric neutrinos can be obtained only with a bigger array. 

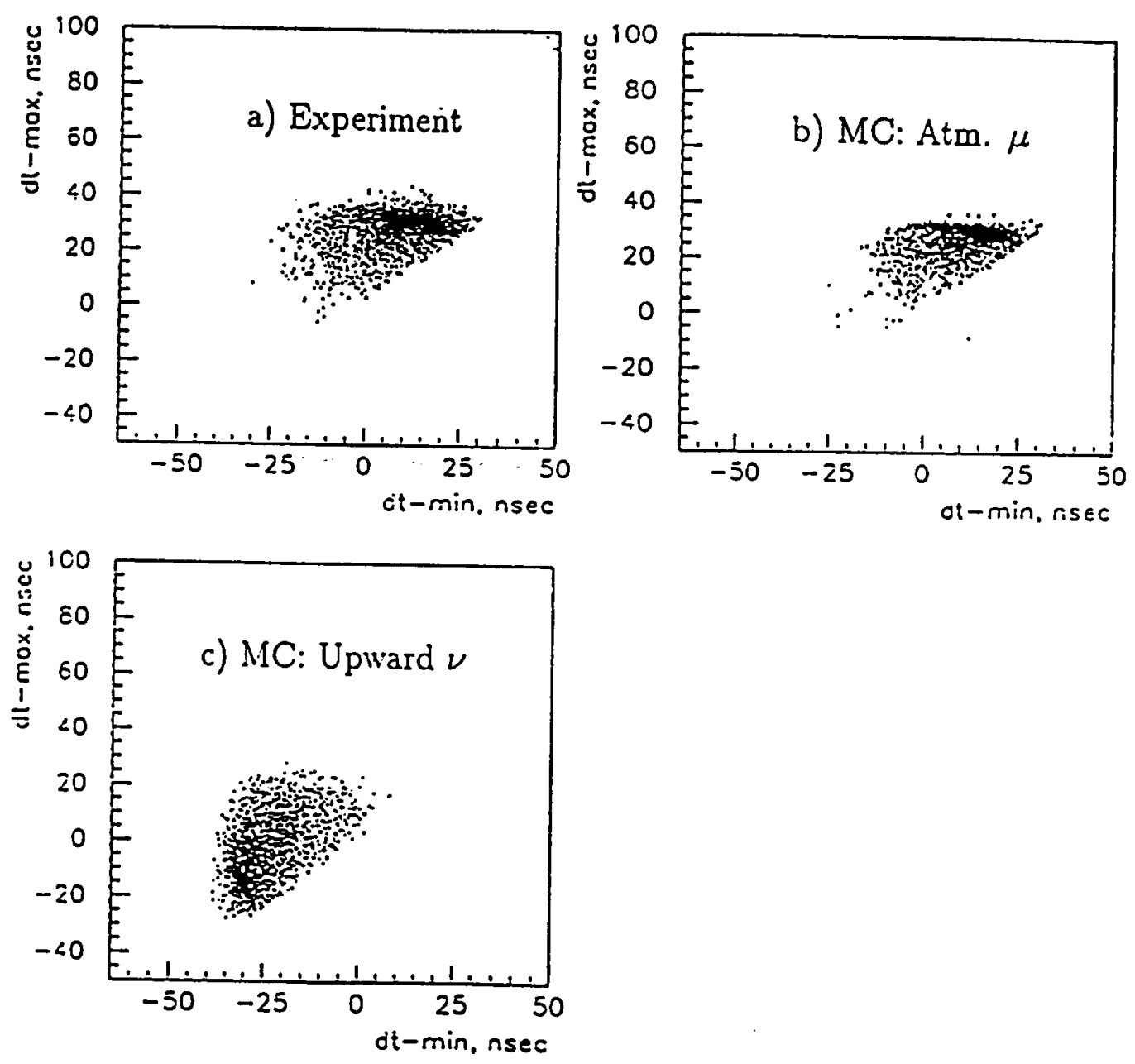

Fig.9: $d t_{\max }$ vs. $d t_{\min }($ see $t$ ext) for: a) NT-36 data, b) atmospheric muons (MC) and c) upward muons from neutrino interactions (MC). The event sample plotted has been fitted and cleaned by the quality criteria described in the text. 


\section{Conclusions}

The results from NT-36 and their agreement with MC calculations support our confidence based on simulations of NT-200, namely, that an underwater array consisting of 200 PMTs at $1 \mathrm{~km}$ depth can be operated as a neutrino telescope. The necessary cuts for background rejection will reduce the neutrino signal by a factor 2 only. This result contradicts concerns [29] that the cuts for background rejection may have to be so severe that they make the detector an order of magnitude less sensitive than if it would be deployed in $4 \mathrm{~km}$ depth.

At the time of writing this paper, the first stages of DUMAND-II [15] and AMANDA [19] are going to be mounted. Together with an upgraded BAIKAL detector scheduled to be deployed in March/April 1994, the next year might turn the tide and allow the first identification of neutrino generated events in deep underwater detectors.

\section{Acknowledgements}

The experimental data used in section 4 have been obtained in a combined effort of all the members of our collaboration. We are indepted to N.M.Budnev, G.V.Domogatzky L.A.Kuzmichev and Yu.V.Parfenov and P.Söding for helpful discussions. Two of us (C.S and R.W.) enjoyed the excellently organized 3rd NESTOR workshop. We thank Leonidas Resvanis and his tireless team.

\section{$7 \quad$ References}

1. L.B.Bezrukov et al., Proc 2nd Int. Symp. Underground Physics Moscow (1988) 230

2. I.A.Belolaptikov et al., Proc. XVII Int. Conf. on High En. Physics, Dallas (1992) 1246

3. I.A.Belolaptikov et al., Proc. 5th Int. Symposium on Neutrino Telescopes, Venice (1993) 
4. J.Babson et al., Phys.Rev.D, 42 (1990) 3613

5. L.Resvanis, Proc. High Energy Neutrino Astrophysics Workshop, Hawaii 1992

6. L.Resvanis, Proc. 5th Int. Symposium on Neutrino Telescopes, Venice (1993) 321

7. I.A.Belolaptikov et al., BAIKAL Note 93-06, to be published in Proc. 3rd TAUP Workshop 1993 (Gran Sasso)

8. See the talk of $R$. Wischnewski at this conference

9. I.A.Belolaptikov et al., Proc. 3rd Int. Workshop on Neutrino telescopes, Padova (1991) 365

10. S.D.Alatin et al., Nucl.Phys.B (Proc.Suppl.) 28A (1992) 491

11. I.Sokalski, Ch.Spiering (eds.), The Baikal Neutrino Telescope NT-200 (Project Description), Baikal Notc 92-03

12. I.A.Belolaptikov et al., Proc. 23rd ICRC, Calgary 1993, 573

13. DUMAND-II proposal, HDC-2-88, Hawaii 1988

14. C.M.Alexander et al., Proc. 23rd ICRC, Calgary 1993, 515

15. see the talk of $P$. Grieder at this conference

16. D.Lowder et al., AMANDA, proposal to NSF, 1992

17. S.Tilav et al., Proc. 23rd ICRC, Calgary 1993, 561

18. P.O.Hulth, talk to be published in Proc. 3rd TAUP Workshop, Gran Sasso 1993

19. See the talk of B. Erlangtsson at this conference

20. V.J.Stenger, private communication

21. Th.Mikolajski, Diploma Thesis, Zeuthen 1991

22. A.Okada, ICRR Report 209-90-2, Tokyo 1990

23. M.D.Galperin and S.I.Klimushin, INR Internal Report, Moscow 1989 (in russian)

24. V.J.Stenger, DUMAND report HDC-1-90, Hawaii 1990

25. A.Okada and K.O'Brien, Proc. 23rd ICRC, Calgary, 523

26. V.J.Stenger, DUMAND Internal Report DIR-91-16, Hawaii 1991

27. I.A.Belolaptikov et al., Proc 23rd ICRC, Calgary 1993, 577

28. N. Hasselmann, R.Wischnewski, BAIKAL Note 93-12, Zeuthen 1993

29. V.J.Stenger, Proc. 2nd NESTOR Workshop (Pylos 1992) 79

30. S.N.Bosiev et al., INR-Preprint P-0630, Moscow 1989 